\title{
Status of the TORCH time-of-flight detector
}

\section{T. Hadavizadeh ${ }^{1 *}$, S. Bhasin ${ }^{2}$, T. Blake ${ }^{3}$, N. Brook ${ }^{4}$, M. F. Cicala ${ }^{3}$, T. Conneely $^{5}$, D.

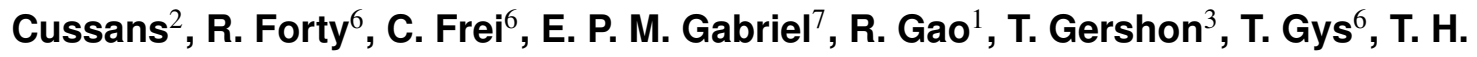 Hancock $^{1}$, N. Harnew ${ }^{1}$, M. Kreps ${ }^{3}$, J. Milnes ${ }^{5}$, D. Piedigrossi ${ }^{6}$, J. Rademacker ${ }^{2}$, M. van Dijk ${ }^{8}$}

${ }^{1}$ Denys Wilkinson Laboratory, University of Oxford, Keble Road, Oxford OXI 3RH, UK

${ }^{2}$ H.H. Wills Physics Laboratory, University of Bristol, Tyndall Avenue, Bristol BS8 ITL, UK

${ }^{3}$ Department of Physics, University of Warwick, Coventry, CV4 7AL, UK

${ }^{4}$ University of Bath, Claverton Down, Bath BA2 7AY, UK

${ }^{5}$ Photek Ltd., 26 Castleham Road, St Leonards on Sea, East Sussex, TN38 9NS, UK

${ }^{6}$ CERN, EP Department, CH 1211, Geneva 23, Switzerland

${ }^{7}$ School of Physics and Astronomy, University of Edinburgh, James Clerk Maxwell Building, Edinburgh EH9 3FD, UK

${ }^{8}$ CERN, EN Department, CH 1211, Geneva 23, Switzerland

E-mail: tom.hadavizadehecern.ch

TORCH is a novel time-of-flight detector, designed to provide $\pi / \mathrm{K}$ particle identification up to $10 \mathrm{GeV} / c$ momentum over a $10 \mathrm{~m}$ flight path. Based on the DIRC principle, Cherenkov photons are produced in a quartz plate of $10 \mathrm{~mm}$ thickness, where they propagate to the periphery of the plate by total-internal reflection. There the photons are focused onto an array of micro-channel plate photomultipliers (MCP-PMTs) which measure their arrival times and spatial positions. A time resolution of 70 ps per detected Cherenkov photon is expected, which results in a time-offlight resolution of $15 \mathrm{ps}$, given typically 30 detected photons per track. For a future application, a full-scale TORCH detector has been proposed for the future LHCb upgrade, which comprises 18 modules with 198 MCP-PMTs. To demonstrate the TORCH principle, a half-height $(1250 \times$ $660 \times 10 \mathrm{~mm}^{3}$ ) prototype module has been tested in a $8 \mathrm{GeV} / c$ mixed proton-pion beam at the CERN PS. Customised $53 \times 53 \mathrm{~mm}^{2}$ MCP-PMTs of effective granularity $128 \times 8$ pixels have been employed, which have been developed in collaboration with an industrial partner. The singlephoton timing performance and photon yields have been measured and are close to specification, demonstrating the TORCH concept.

European Physical Society Conference on High Energy Physics - EPS-HEP2019 -

10-17 July, 2019

Ghent, Belgium

* Speaker and Corresponding author. 


\section{Introduction}

The TORCH (Time of Internally Reflected CHerenkov light) detector is a novel low-momentum particle identification (PID) detector that exploits time-of-flight (ToF) to provide positive hadron identification [1,2]. TORCH aims to provide $\pi / K$ separation up to a momentum of $\sim 10 \mathrm{GeV} / c$ and proton identification up to $\sim 20 \mathrm{GeV} / c$. Prompt Cherenkov radiation emitted by charged hadrons in a highly polished quartz plate is collected via total internal reflection. Single photons are focused onto a photon detector plane, where the spatial positions and arrival times are recorded, and used to determine the track species. The difference in the ToF of $10 \mathrm{GeV} / c$ kaons and pions when travelling a flight path of $10 \mathrm{~m}$ is approximately $35 \mathrm{ps}$. Therefore TORCH aims to achieve a time resolution of $10-15$ ps per track, corresponding to a single-photon time resolution of $70 \mathrm{ps}$ for 30 collected photons.
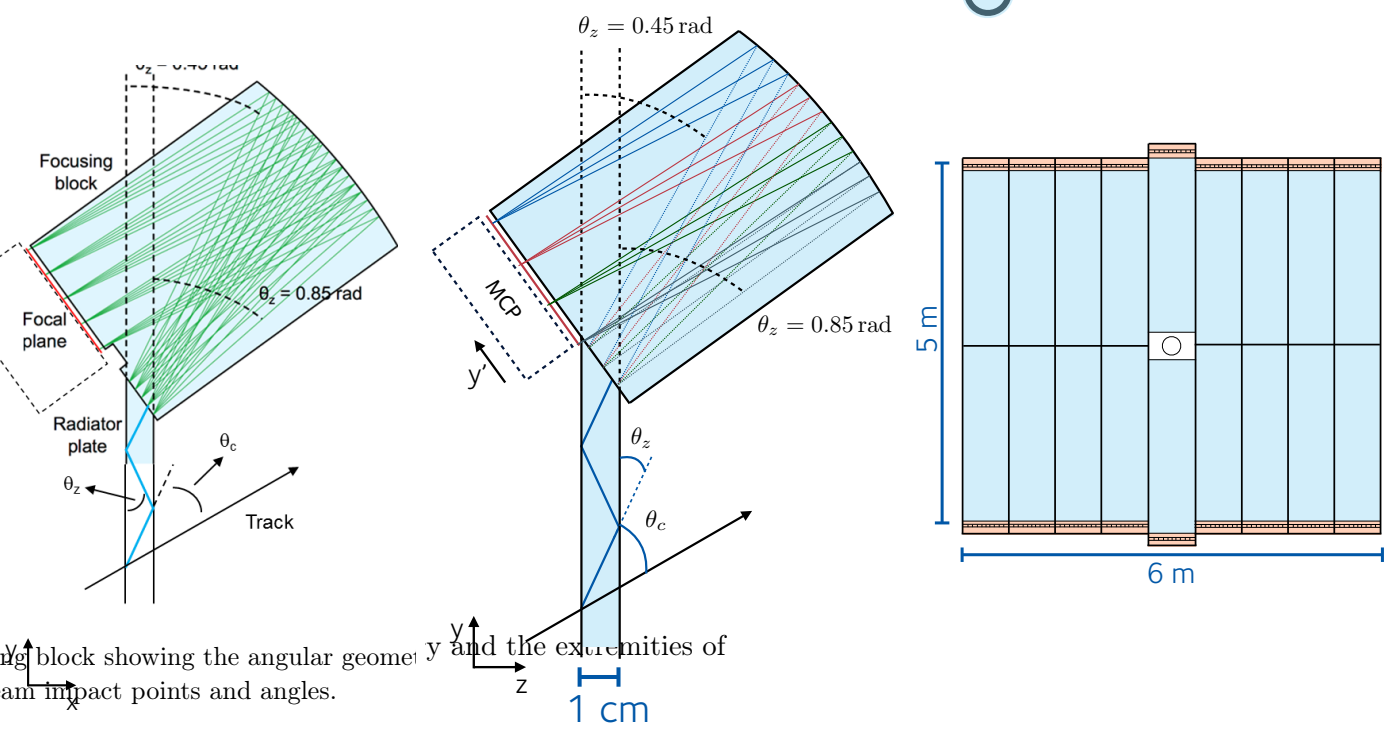

Figure 1: Schematics of the TORCH detector design. (Left) a single TORCH module, (middle) the focusing block, which translates the photon's angle of exit from the quartz plate in the $y z$ plane $\left(\theta_{z}\right)$ into the $y$ ' position on the MCP-PMT detector plane, and (right) the 18-module TORCH detector proposed for LHCb.

\section{Design}

The TORCH detector has been proposed for the upgraded LHCb experiment [3] and consists of 18 quartz modules, spanning an area of $5 \times 6 \mathrm{~m}^{2}$, as shown in Fig. 1. Each module is made up of a quartz plate $\left(2500 \times 660 \times 10 \mathrm{~mm}^{3}\right)$ with a focusing block at either the top or bottom edge. The focusing block, with a cylindrical mirrored surface, translates the angle of exit from the quartz plate in the $y z$ plane to the $y^{\prime}$ vertical position on the photon detector. TORCH could be installed during the LHC Long Shutdown 3 for operation during Run 4 and beyond, and provide complementary PID information to the existing Ring-Imaging Cherenkov (RICH) detectors [4].

Two TORCH prototypes have been developed and tested at the CERN Proton Synchrotron in a $5-8 \mathrm{GeV} p / \pi$ beam. The first, named "Mini-TORCH", is a small-scale module with a $350 \times$ 
$120 \times 10 \mathrm{~mm}^{3}$ radiator plate, instrumented with a single micro-channel plate photomultiplier tube (MCP-PMT). The performance of this prototype has been reported in Refs. [5] and [6]. The second prototype, named "Proto-TORCH", is a half-height, full-width module $\left(1250 \times 660 \times 10 \mathrm{~mm}^{3}\right)$ instrumented with two MCP-PMTs.

\section{Micro-channel Plate PMTs}

Cherenkov radiation is focused onto a plane of MCP-PMTs, developed by industrial partner Photek UK (Ltd) [7]. Each MCP-PMT, shown in Fig. 2, has a granularity of $64 \times 64$ pixels over a $53 \times 53 \mathrm{~mm}^{2}$ active area. The horizontal (non-focusing) channels are grouped electronically and the device is read out with a granularity of $64 \times 8$. The MCP-PMTs use charge sharing to improve the granularity in the vertical (focusing) direction, giving $128 \times 8$ effective pixelisation. The avalanche of charge produced by a single photon in the MCP typically induces hits on multiple anode pixels. The centroid of these hits allows the spatial and timing precision of the photon arrival time to be improved. The MCP-PMTs are designed to withstand an integrated charge of $5 \mathrm{C} \mathrm{cm}^{-2}$ by utilising an atomic layer deposition (ALD) coating onto the MCPs. The devices are read out with customised electronics [8] that use the NINO and HPTDC chipsets developed for the ALICE TOF detector $[9,10]$. The NINO is a discriminator that provides time-over-threshold information, which is used to correct the timing for the pulse-height-dependent signal shape (time-walk). Additionally, corrections are applied for non-linearities in the HPTDC binning. Time reference pulses from an external ToF system [2] are injected into eight channels per MCP-PMT.
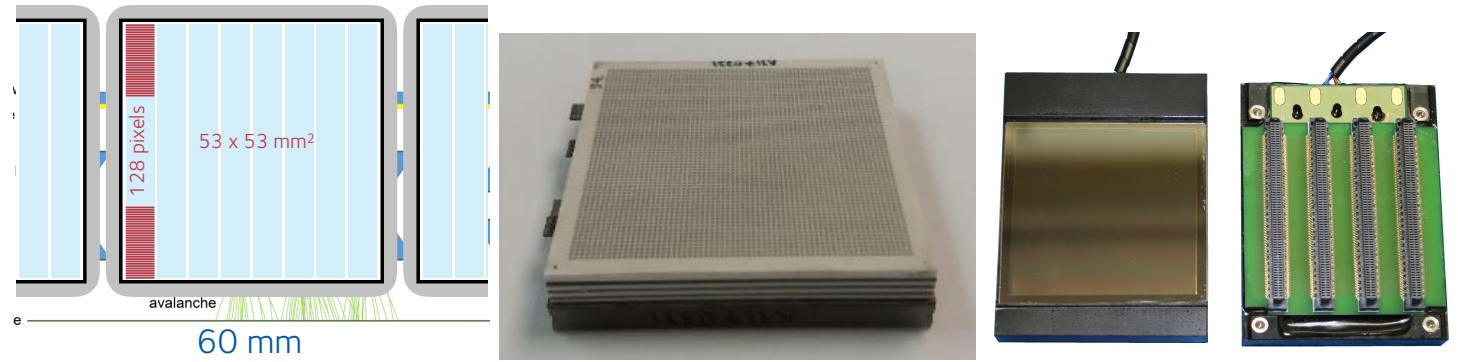

Figure 2: (Left) Schematic of the MCP-PMT pixel layout, (middle) a photograph of the MCP-PMT surface, and (right) the MCP-PMT connectors.

\section{Beam tests analysis}

The Proto-TORCH module was tested in a $8 \mathrm{GeV} p / \pi$ beam in October 2018. The module was instrumented with two MCP-PMTs, corresponding to 1024 readout channels, of which 16 were time reference channels. An example of the spatial distribution of hits is shown in Fig. 3. The cones of Cherenkov radiation emitted by the charged hadrons are focused into a hyperbolelike pattern, which are folded in on themselves when the photons are reflected off the sides of the module. The two MCP-PMTs had differing quantum efficiencies, leading to the different numbers of hits as seen in the figure. The number of dead pixels decreases from the left-hand to right-hand 

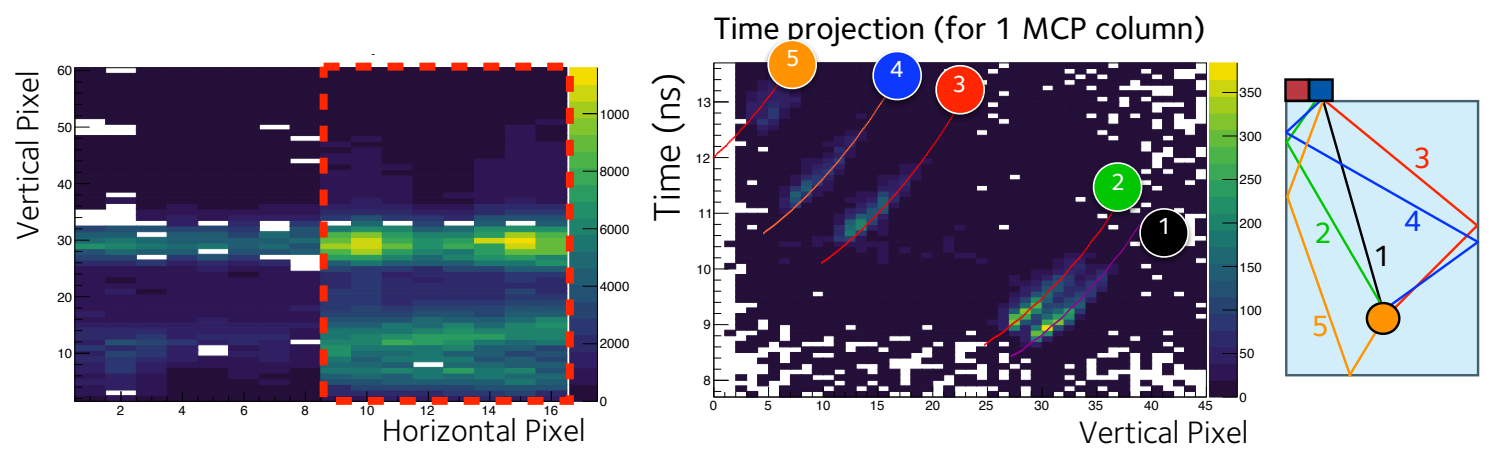

Figure 3: (Left) The spatial distribution of hits on the MCP-PMT as measured in the beam test. The MCPPMT used in the subsequent analysis is highlighted. (Middle) The time projection of a single MCP-PMT column, with the different photon trajectories labelled as shown in the diagram on the right.

side MCP-PMT as a result of improvements in the NINO wire bonding procedure. The test beam analysis described below concentrates on the MCP-PMT with the higher quantum efficiency.

Due to the charge sharing in the MCP-PMT, single photons result in hits on multiple pixels. Hits on a single MCP-PMT column but on adjacent vertical channels are combined by a clustering algorithm to create a single photon cluster if the hits arrive within $1 \mathrm{~ns}$ on adjacent pixels. The clustered spatial hit-maps are extended to create time-projection plots, in which the hits in the focusing plane ( $\left.y^{\prime}\right)$ are projected along the time axis. An example is shown for a single MCP-PMT column in Fig. 3, along with reconstructed predictions calculated from the incident track position and angle. It can be seen that the different orders of side reflection are cleanly separated, and the widths of each order are measured to determine the single-photon time resolution.

\subsection{Time resolution}

The time resolution is determined using simultaneous unbinned maximum-likelihood fits to the photon time of arrival with respect to the time reference. The time resolution is corrected for the uncertainty arising from the time reference pulse (43.2 ps) and from the beam spread (15.1$30.4 \mathrm{ps}$, depending on the distance between the beam and the MCP-PMT plane). A separate fit model is created for each pixel, comprising a polynomial background distribution and peaking structures modelled by Crystal Ball functions. The TORCH reconstruction predictions are used to determine how many peaking structures are expected for each pixel. Additionally, background shapes are included to account for structures observed in the data, namely bands across all pixels that appear at the same time, likely to be a result of scattered beam-related light.

The time resolution is determined for different beam positions in vertical height. The distance from the MCP-PMT plane to the beam is varied from $17.5 \mathrm{~cm}$ to $101.0 \mathrm{~cm}$ in four steps. The results of the fits for each MCP-PMT column and beam position is shown in Fig. 4, corresponding to all photon clusters that contain between 2 and 5 hits. Additionally, the time resolution is measured as a function of the number of hits per cluster, as shown in Fig. 4. The spread in the photon arrival time reduces as the size of the cluster increases. It can be seen that the time resolution of the ProtoTORCH demonstrator is in the range 60-130 ps, approaching or exceeding the design goal of $70 \mathrm{ps}$ per photon for specific beam positions and cluster sizes. 

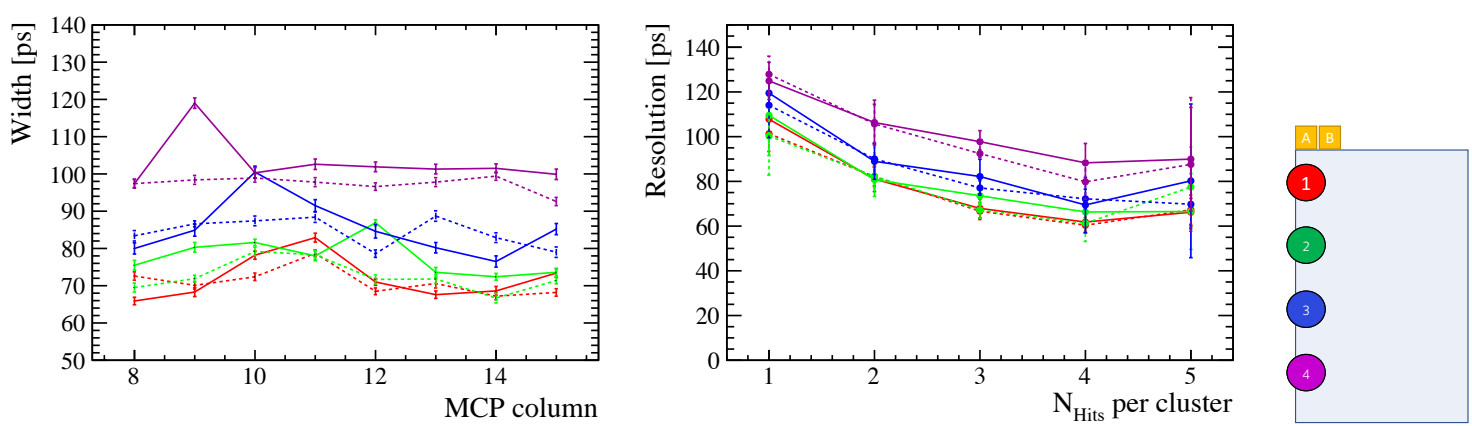

Figure 4: (Left) Time resolution as determined for different MCP-PMT columns and beam positions, as indicated in the diagram on the right. (Middle) Variation in the time resolution as a function of the number of hits per cluster, for the different beam positions.

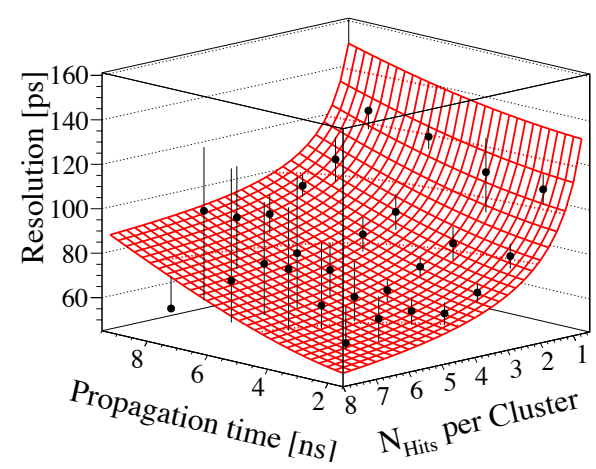

Figure 5: The TORCH time resolution displayed as a function of the propagation time and number of hits per cluster. The overlaid surface is the result of the $2 \mathrm{D}$ fit described in the text.

A two dimensional fit has been performed to the time resolutions as a function of the beam position and cluster size to quantify the contributions from different sources, as shown in Fig. 5. The TORCH time resolution, $\sigma_{\mathrm{TORCH}}$, is assumed to vary according to the expression

$$
\sigma_{\mathrm{TORCH}}^{2}=\sigma_{\mathrm{const}}^{2}+\sigma_{\text {prop }}\left(t_{P}\right)^{2}+\sigma_{\mathrm{RO}}\left(N_{\mathrm{Hits}}\right)^{2},
$$

where the constant contribution, $\sigma_{\text {const }}$, is attributed to sources such as the intrinsic MCP-PMT time resolution; the term $\sigma_{\text {prop }}\left(t_{P}\right)$ characterises the contributions that grow with photon propagation time, $t_{P}$, such as dispersive effects; and finally the term $\sigma_{\mathrm{RO}}\left(N_{\mathrm{Hits}}\right)$ that is a function of the number of hits in a cluster, $N_{\text {Hits. }}$. The two-dimensional fit determines the separate components to be

$$
\begin{gathered}
\sigma_{\text {const }}=33.0 \pm 7.1 \mathrm{ps}, \\
\sigma_{\text {prop }}\left(t_{P}\right)=(7.8 \pm 0.7) \times 10^{-3} \times t_{P} \mathrm{ps}, \text { and } \\
\sigma_{\mathrm{RO}}\left(N_{\text {Hits }}\right)=\frac{100.5 \pm 5.7}{\sqrt{N_{\text {Hits }}}} \mathrm{ps}
\end{gathered}
$$

where a linear dependence on $t_{P}$ and a $1 / \sqrt{N}$ dependence on $N_{\text {Hits }}$ has been assumed. The sources of these uncertainties are currently under study in laboratory tests. Further electronics calibrations are expected to improve the latter two resolution contributions. 


\subsection{Photon yields}

The desired TORCH per-track time resolution assumes that 30 photons per charged track are collected. The number of photons clusters in the Proto-TORCH demonstrator are counted and compared to the number obtained in Monte Carlo. The TORCH prototype is simulated using GEANT4 to model the optical processes [11], whilst custom libraries are used to model the detector and electronics read-out responses. Various sources of inefficiency are accounted for, including the surface roughness of the quartz and the MCP-PMT quantum and collection efficiencies. The distributions of photon yields are shown in Fig. 6. Reasonable agreement is seen when the beam is close to the MCP-PMT, but discrepancies are observed when the beam is positioned further down the radiator plate. The source of these discrepancies are still under study. As the current prototype is instrumented with only 2 MCP-PMTs out of a posible total of 11, the light yield is expected to improve by a factor of 5.5 in a fully instrumented module. Additionally, the two MCP-PMTs under test had peak quantum efficiencies of only around $13.1 \%$ and $17.5 \%$, whilst the final MCP-PMTs are expected to have peak quantum efficiencies of at least $25 \%$, further increasing the photon yield.
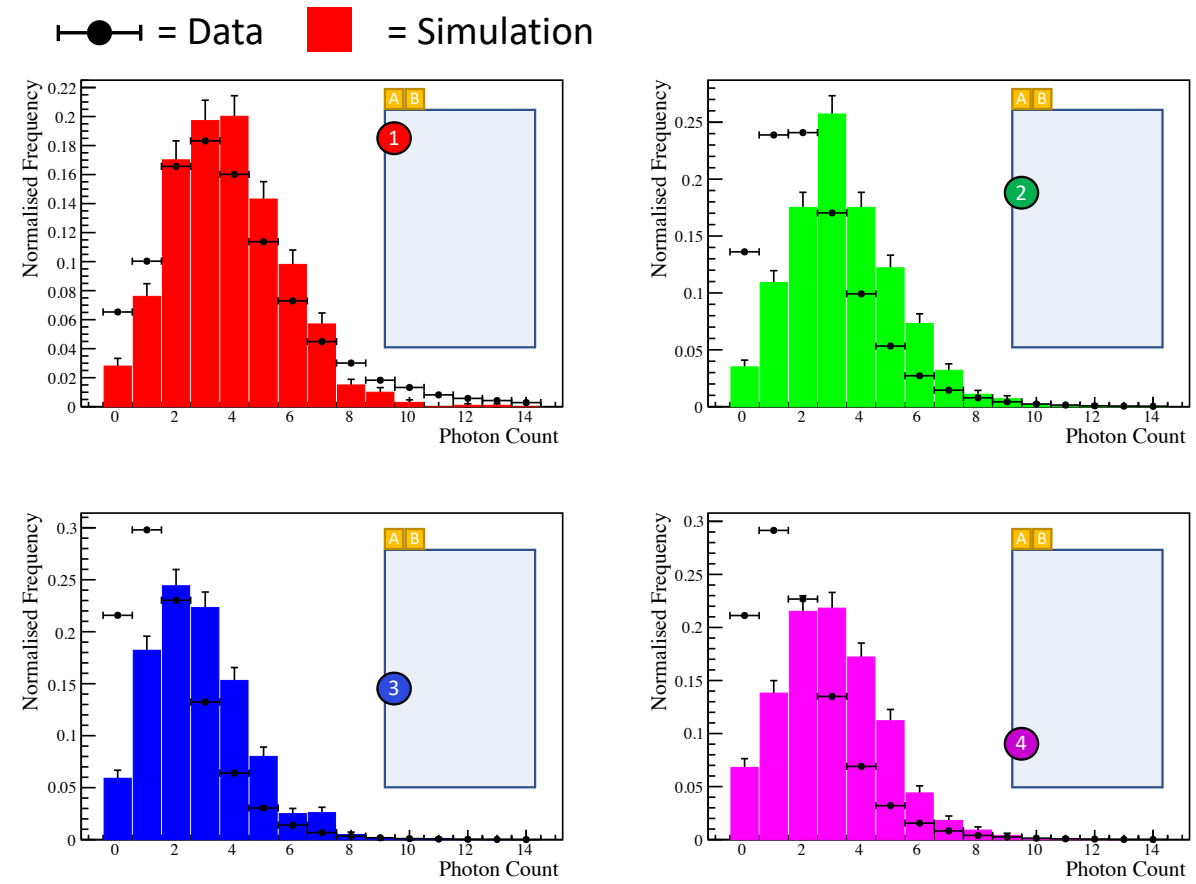

Figure 6: Photon yields in data and simulation when the beam is positioned $17.5 \mathrm{~cm}$ (top left), $45.3 \mathrm{~cm}$ (top right), $73.2 \mathrm{~cm}$ (bottom left) and $101.0 \mathrm{~cm}$ (bottom right) from the MCP-PMT.

\section{Simulation and physics studies}

The full TORCH detector, comprised of 18 modules and 198 MCP-PMTs, has been simulated within the framework of LHCb experiment [12]. The optical processes are modeled in GEANT4 [11] and the sources of inefficiency described in Section 4.2 are taken into account. The PID performance is determined for the LHCb upgrade (Run 4) conditions in which the instantaneous luminosity is expected to be $\mathscr{L}=2 \times 10^{33} \mathrm{~cm}^{-2} \mathrm{~s}^{-1}$ [13]. A PID algorithm compares the 
likelihood of each charged track hypothesis by generating the expected photon patterns in the event, in a similar procedure to that used in the LHCb RICH reconstruction [14]. The PID performance shown in Fig. 7 demonstrates that the TORCH detector has excellent separation power in the range up to $10 \mathrm{GeV} / c$ for kaons and pions, and up to $20 \mathrm{GeV} / c$ for kaons and protons.
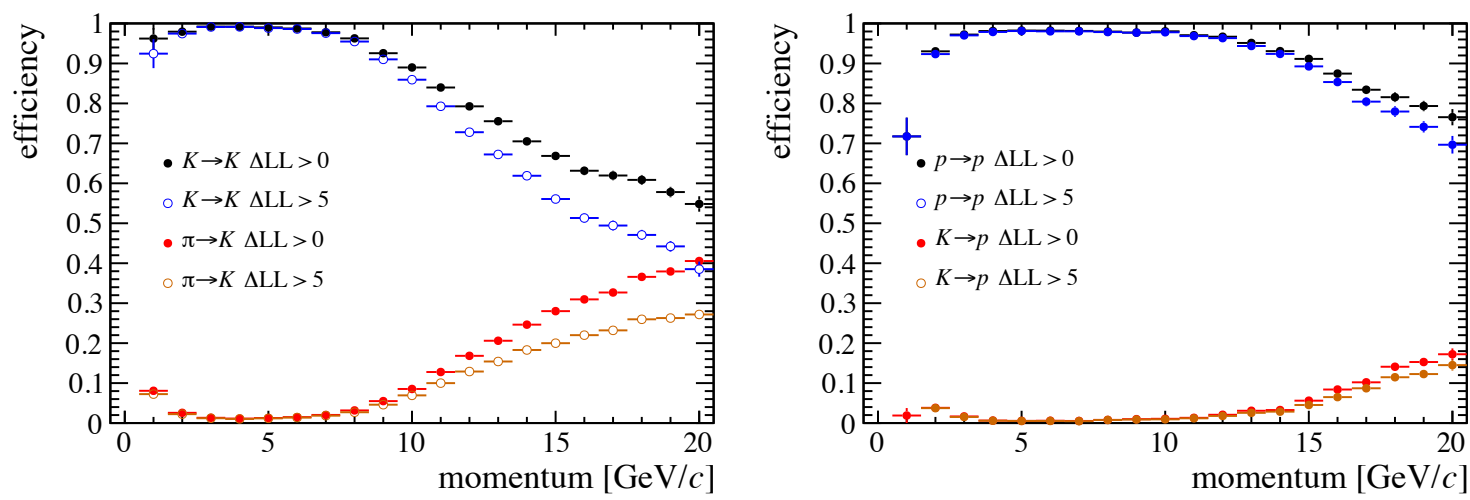

Figure 7: TORCH PID performance of the full TORCH detector as determined from simulations. (Left) kaon-pion separation and (right) kaon-proton separation.

This PID performance is used to quantify the benefits of TORCH to the LHCb experiment in selected physics channels. As expected, modes with low-momentum kaons and protons benefit the most. The modes studied include semi-leptionic processes such as $\Lambda_{b}^{0} \rightarrow p \mu^{-} \bar{v}_{\mu}$ and $B_{s}^{0} \rightarrow K^{-} \mu^{+} \nu_{\mu}$, and baryonic modes including $\Lambda_{b}^{0} \rightarrow J / \psi p K^{-}$and $\Lambda_{b}^{0} \rightarrow p \pi^{-} \pi^{+} \pi^{-}$. The TORCH detector is found to significantly improve the signal efficiency, aid in the rejection of misidentified backgrounds, and reduce the phase-space dependence of PID efficiencies in these modes. The increase in signal efficiency varies from $10 \%$ to more than 50\% depending on the kinematics of the mode. Additionally, TORCH is found to benefit the efficiency of flavour tagging algorithms, utilised in many measurements. Some flavour tagging algorithms rely on identifying low momentum kaons and protons; the addition of TORCH PID improves the effective tagging power by around $25 \%$.

\section{Conclusions and future plans}

In conclusion, a successful series of beam tests has been conducted, the latest with the ProtoTORCH module. The single photon time resolution has been measured in the range 60-130 ps, achieving the goal of $70 \mathrm{ps}$ in some configurations. Further laboratory tests are ongoing to independently verify the contributions to the time resolution, and improved electronics calibrations are expected to further enhance the performance. A full-scale TORCH detector has been simulated in the LHCb experiment and studies indicate significant improvements to the LHCb physics potential. A fully-instrumented TORCH module with 11 MCP-PMTs will be tested in the future.

\section{Acknowledgements}

The support is acknowledged of the Science and Technology Research Council, UK, grant 
number ST/P002692/1, and of the European Research Council through an FP7 Advanced Grant (ERC-2011-AdG299175-TORCH).

\section{References}

[1] M. J. Charles and R. Forty, TORCH: Time of Flight Identification with Cherenkov Radiation, Nucl. Instrum. Meth. A639 (2011) 173 [1009.3793].

[2] N. Brook et al., Testbeam studies of a TORCH prototype detector, Nucl. Instrum. Meth. A908 (2018) $256[1805.04849]$.

[3] LHCB collaboration, Physics case for an LHCb Upgrade II - Opportunities in flavour physics, and beyond, in the HL-LHC era, 1808.08865.

[4] LHCB collaboration, LHCb RICH: Technical Design Report, 2000.

[5] N. Harnew et al., Status of the TORCH time-of-flight project, 1812.09773.

[6] N. Harnew et al., TORCH: a large area time-of-fight detector for particle identification, Nucl. Instrum. Meth. A936 (2019) 595 [1810.06658].

[7] J. S. Milnes, T. M. Conneely, J. S. Lapington, P. Kapetanopoulos, C. Slatter and J. Howorth, The TORCH PMT, a close packing, multi-anode, long life MCP-PMT for Cherenkov applications, Nucl. Instrum. Meth. A766 (2014) 183.

[8] R. Gao et al., Development of TORCH readout electronics for customised MCPs, JINST 11 (2016) C04012.

[9] A. N. Akindinov et al., Design aspects and prototype test of a very precise TDC system implemented for the multigap RPC of the ALICE-TOF, Nucl. Instrum. Meth. A533 (2004) 178.

[10] F. Anghinolfi, P. Jarron, A. N. Martemyanov, E. Usenko, H. Wenninger, M. C. S. Williams et al., NINO: An ultra-fast and low-power front-end amplifier/discriminator ASIC designed for the multigap resistive plate chamber, Nucl. Instrum. Meth. A533 (2004) 183.

[11] J. Allison et al., Recent developments in Geant4, Nucl. Instrum. Meth. A835 (2016) 186.

[12] LHCB collaboration, The LHCb simulation application, Gauss: Design, evolution and experience, J. Phys. Conf. Ser. 331 (2011) 032023.

[13] LHCB collaboration, Framework TDR for the LHCb Upgrade: Technical Design Report, Tech. Rep. CERN-LHCC-2012-007. LHCb-TDR-12, Apr, 2012.

[14] R. Forty, RICH pattern recognition for LHCb, Nucl. Instrum. Meth. A433 (1999) 257. 